\title{
Catalytic oxidation using nitrous oxide
}

\author{
Beltran-Prieto Juan Carlos ${ }^{1, *}$, Kolomaznik Karel $^{1}$ \\ ${ }^{1}$ Faculty of Applied Informatics, Tomas Bata University in Zlín, nám. T. G. Masaryka 5555, 76001 Zlín, Czech Republic
}

\begin{abstract}
Nitrous oxide is a very inert gas used generally as oxidant as it offers some advantage compared with other oxidants such as $\mathrm{O}_{2}$ but a considerably higher temperature $\left(>526{ }^{\circ} \mathrm{C}\right)$ is often required. For particular cases such as the oxidation of sugar alcohols, especially for the oxidation of primary alcohols to aldehydes, $\mathrm{N}_{2} \mathrm{O}$ has the advantage over $\mathrm{O}_{2}$ of a higher reaction selectivity. In the present paper we present the modelling of oxidation reaction of sugar alcohols using an oxidizing agent in low concentrations, which is important to suppress subsequent oxidation reactions due to the very low residual concentrations of the oxidizing agent. For orientation experiments we chose nitrous oxide generated by thermal decomposition of ammonium nitrate. Kinetic modeling of the reaction was performed after determination of the differential equations that describe the system under study.
\end{abstract}

\section{Introduction}

In heterogenous catalysis, several metal oxides have been extensively used in chemical reactions. Among the most effective catalysts are iron-containing acidic zeolites which yield surface-activated iron-oxo species ( $\alpha$-Oxygen). Ohtani reported that the oxidation of organic compounds at room temperature can be performed by the reductive decomposition of $\mathrm{N}_{2} \mathrm{O}$ to produce hydroxyl radical $(\cdot \mathrm{OH})$ selectively, however it is observed only under limited reaction conditions, such as $\gamma$-radiolysis, photolisis or electrolisis [1]. $\mathrm{N}_{2} \mathrm{O}$ is used as a trap for hydrated electron as well as hydroxyl radical $(\cdot \mathrm{OH})$ generated by $\gamma$-radiolysis of water via reactions (1) and (2)

$$
\begin{aligned}
& \mathrm{H}_{2} \mathrm{O} \stackrel{\text { r ray }}{\longrightarrow}: \mathrm{OH}+e^{-}{ }_{a q}+\mathrm{H}^{+} \\
& \mathrm{N}_{2} \mathrm{O}+e^{-} a q+\mathrm{H}_{2} \mathrm{O} \rightarrow \mathrm{N}_{2}+: \mathrm{OH}+\mathrm{OH}^{-}
\end{aligned}
$$

The oxidation kinetics is based on the idea of the reaction mechanism, quantitative description of the dependences of the reaction rates on the concentration of the reacting components. The description is realized by vector differential equation, the solutions of which are time dependent of the concentrations of the oxidation intermediate products. [2]-[6] Generally, chemical reactions are accompanied by various relatively complex simultaneous reactions resulting in a complicated blend of intermediate products. As a general model, the oxidation reaction of sugar alcohols (S) using nitrous oxide (N) for the production of derivatives (A) and (B) in isothermal conditions is discussed next. The diagram of the system is presented in Fig. 1 having three inputs $\left(F, C_{N 0}\right.$, and $\left.C_{S 0}\right)$, four state variables $(A, N, S$ and $B)$ and four output variables $(A, N, S$, and $B)$.

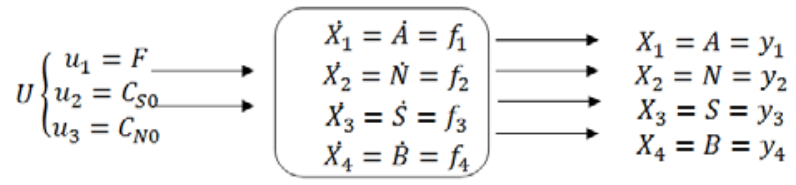

Fig. 1. Scheme of the oxidation of glycerol with $\mathrm{NO}_{2}$ describing the input, state variable and output

\section{Mathematical model of oxidation with $\mathrm{NO}_{2}$}

The set of equations that describe the mass balance, including of chemical reactions in which are supposed to be of the first order mechanism, of the components is presented in (3) to (6)

$$
\begin{aligned}
\frac{\mathrm{dA}}{\mathrm{dt}} & =k_{1} \cdot S \cdot N-k_{2} \cdot A \cdot N-\frac{F}{V} A \\
\frac{\mathrm{dN}}{\mathrm{dt}}= & -\mathrm{k}_{1} \cdot \mathrm{S} \cdot \mathrm{N}-\mathrm{k}_{2} \cdot \mathrm{A} \cdot \mathrm{N}+\frac{\mathrm{F}}{\mathrm{V}} \mathrm{C}_{\mathrm{N} 0}-\frac{\mathrm{F}}{\mathrm{V}} \mathrm{N} \\
\frac{\mathrm{dS}}{\mathrm{dt}} & =-\mathrm{k}_{1} \cdot \mathrm{S} \cdot \mathrm{N}+\frac{\mathrm{F}}{\mathrm{V}} \mathrm{C}_{\mathrm{S} 0}-\frac{\mathrm{F}}{\mathrm{V}} \mathrm{S} \\
\frac{\mathrm{dB}}{\mathrm{dt}} & =\mathrm{k}_{2} \cdot \mathrm{A} \cdot \mathrm{N}-\frac{\mathrm{F}}{\mathrm{V}} \mathrm{B}
\end{aligned}
$$

The described system is non-linear. The elements of matrix A, B, are the following [7]-[9]:

$$
\begin{aligned}
& a_{11}=\frac{\partial f_{1}{ }^{0}}{\partial A}=-k_{2} N^{0}-\frac{F^{0}}{V} ; a_{12}=\frac{\partial f_{1}{ }^{0}}{\partial N}=k_{1} S^{0}- \\
& k_{2} A^{0} ; a_{13}=\frac{\partial f_{1}{ }^{0}}{\partial S}=k_{1} N^{0} ; a_{14}=\frac{\partial f_{1}{ }^{0}}{\partial B}=0 \\
& a_{21}=\frac{\partial f_{2}{ }^{0}}{\partial A}=-k_{2} N^{0} ; a_{22}=\frac{\partial f_{2}{ }^{0}}{\partial N}=-k_{1} S^{0}-k_{2} A^{0}- \\
& \frac{F^{0}}{V} ; a_{23} \frac{\partial f_{2}{ }^{0}}{\partial S}=-k_{1} N^{0} ; a_{24}=\frac{\partial f_{2}{ }^{0}}{\partial B}=0 \\
& a_{31}=\frac{\partial f_{3}{ }^{0}}{\partial A}=0 ; a_{32}=\frac{\partial f_{3}{ }^{0}}{\partial N}=-k_{1} S^{0} ; a_{33}=\frac{\partial f_{3}{ }^{0}}{\partial S}= \\
& -k_{1} N^{0}-\frac{F^{0}}{V} ; a_{34}=\frac{\partial f_{3}{ }^{0}}{\partial B}=0
\end{aligned}
$$

\footnotetext{
* Corresponding author: prieto@fai.utb.cz
} 
$a_{41}=\frac{\partial f_{4}{ }^{0}}{\partial A}=k_{2} N^{0} ; a_{42}=\frac{\partial f_{4}{ }^{0}}{\partial N}=k_{2} A^{0} ; a_{43}=\frac{\partial f_{4}{ }^{0}}{\partial S}=$ $0 ; a_{44}=\frac{\partial f_{4}{ }^{0}}{\partial B}=-\frac{F^{0}}{V}$

Considering the control variables, equations (11) to (14) are obtained:

$$
\begin{array}{r}
b_{11}=\frac{\partial f_{1}{ }^{0}}{\partial F}=-\frac{A^{0}}{V} ; b_{12}=\frac{\partial f_{1}{ }^{0}}{\partial C_{S 0}}=0 ; b_{13}=\frac{\partial f_{1}{ }^{0}}{\partial C_{N 0}}=0 \\
b_{21}=\frac{\partial f_{2}{ }^{0}}{\partial F}=\frac{\left(C_{\left.N 0^{0}-N^{0}\right)}\right)}{V} b_{22}=\frac{\partial f_{2}{ }^{0}}{\partial C_{S 0}}=0 ; b_{23}=\frac{\partial f_{2}{ }^{0}}{\partial C_{N 0}}=\frac{F^{\circ}}{V} \\
b_{31}=\frac{\partial f_{3}{ }^{0}}{\partial F}=\frac{\left(C_{S 0}-S^{0}\right)}{V} ; b_{32}=\frac{\partial f_{3}{ }^{0}}{\partial C_{S 0}}=\frac{F^{\circ}}{V} ; b_{33}=\frac{\partial f_{3}{ }^{0}}{\partial C_{N 0}}=0 \\
b_{41}=\frac{\partial f_{4}{ }^{0}}{\partial F}=-\frac{B^{0}}{V} ; b_{42}=\frac{\partial f_{4}{ }^{0}}{\partial C_{S 0}}=0 ; b_{43}=\frac{\partial f_{1}{ }^{0}}{\partial C_{N 0}}=0
\end{array}
$$

Therefore, the representation of the state vector is given by:

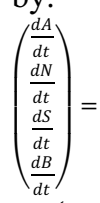

$$
\begin{aligned}
& \left(\begin{array}{cccc}
-\left(k_{2} N^{0}+\frac{F^{0}}{V}\right) & k_{1} S^{0}-k_{2} A^{0} & k_{1} N^{0} & 0 \\
-k_{2} N^{0} & -\left(k_{1} S^{0}+k_{2} A^{0}+\frac{F^{0}}{V}\right) & k_{1} N^{0} & 0 \\
0 & -k_{1} S^{0} & -\left(k_{1} N^{0}+\frac{F^{0}}{V}\right) & 0 \\
k_{2} N^{0} & k_{2} A^{0} & 0 & -\frac{F^{0}}{V}
\end{array}\right)\left(\begin{array}{c}
\Delta A \\
\Delta N \\
\Delta S \\
\Delta B
\end{array}\right)+ \\
& \left(\begin{array}{ccc}
-\frac{A^{0}}{V} & 0 & 0 \\
\frac{\left(C_{N 0}-N^{0}\right)}{V} & 0 & \frac{F^{\circ}}{V} \\
\frac{\left(C_{S 0}-S^{0}\right)}{V} & \frac{F^{0}}{V} & 0 \\
-\frac{B^{0}}{V} & 0 & 0
\end{array}\right)\left(\begin{array}{c}
\Delta F \\
\Delta C_{S 0} \\
\Delta C_{N 0}
\end{array}\right)
\end{aligned}
$$

The multiplication procedure leads to the next group of equations:

$$
\begin{aligned}
& \frac{d \Delta A}{d t}=-\left(k_{2} N^{0}+\frac{F^{\circ}}{V}\right) \Delta A+\left(k_{1} S^{0}-k_{2} A^{0}\right) \Delta N+ \\
& \left(k_{1} N^{0}\right) \Delta S-\frac{A^{0}}{V} \Delta F \\
& \frac{d \Delta N}{d t}=-\left(k_{2} N^{0}\right) \Delta A-\left(k_{1} S^{0}+k_{2} A^{0}+\frac{F^{\circ}}{V}\right) \Delta N+ \\
& \left(k_{1} N^{0}\right) \Delta S+\frac{\left(C_{N 0}-N^{0}\right)}{V} \Delta F+\frac{F^{\circ}}{V} \Delta C_{N 0} \\
& \frac{d \Delta S}{d t}=-\left(k_{1} S^{0}\right) \Delta A-\left(k_{1} N^{0}+\frac{F^{\circ}}{V}\right) \Delta S+\frac{1}{V}\left(C_{S 0}-\right. \\
& \left.S^{0}\right) \Delta F+\frac{F^{\circ}}{V} \Delta C_{S 0} \\
& \frac{d \Delta B}{d t}=\left(k_{2} N^{0}\right) \Delta A+\left(k_{2} A^{0}\right) \Delta N-\left(\frac{F^{\circ}}{V}\right) \Delta B-\left(\frac{B^{0}}{V}\right) \Delta F
\end{aligned}
$$

For the purpose of control, it is necessary to introduce the following dimensionless parameters

$$
\begin{aligned}
& A^{*}=\frac{A}{S^{0}} \therefore A=A^{*} \cdot S^{0} \\
& N^{*}=\frac{N}{N^{0}} \therefore N=N^{*} \cdot N^{0} \\
& S^{*}=\frac{S}{S^{0}} \therefore S=S^{*} \cdot S^{0} \\
& B^{*}=\frac{B}{S^{0}} \therefore B=B^{*} \cdot S^{0} \\
& \quad F^{*}=\frac{F}{F^{0}} \therefore F=F^{*} \cdot F^{0} \\
& C_{S 0}{ }^{*}=\frac{C_{S 0}}{S^{0}} \therefore C_{S 0}=C_{S 0}{ }^{*} \cdot S^{0} \\
& C_{N 0}{ }^{*}=\frac{C_{N 0}}{N^{0}} \therefore C_{N 0}=C_{N 0}{ }^{*} \cdot N^{0}
\end{aligned}
$$

After substitution of dimensionless dependencies, equations (25) to (28) are obtained:

$$
\begin{gathered}
\frac{d \Delta A^{*} \cdot S^{0}}{d t}=-\left(k_{2} N^{0}+\frac{F^{\circ}}{V}\right) \Delta A^{*} \cdot S^{0}+\left(k_{1} S^{0}-\right. \\
\left.k_{2} A^{0}\right) \Delta N^{*} \cdot N^{0}+\left(k_{1} N^{0}\right) \Delta S^{*} \cdot S^{0}-\frac{A^{0}}{V} \Delta F^{*} \cdot F^{0} \\
\frac{d \Delta N^{*} \cdot N^{0}}{d t}=-\left(k_{2} N^{0}\right) \Delta A^{*} \cdot S^{0}-\left(k_{1} S^{0}+k_{2} A^{0}+\right. \\
\left.\frac{F^{\circ}}{V}\right) \Delta N^{*} \cdot N^{0}+\left(k_{1} N^{0}\right) \Delta S^{*} \cdot S^{0}+\frac{\left(C_{N 0}-N^{0}\right)}{V} \Delta F^{*} \cdot F^{0}+ \\
\frac{F^{\circ}}{V} \Delta C_{N 0}{ }^{*} \cdot N^{0} \\
\frac{d \Delta S^{*} \cdot S^{0}}{d t}=-\left(k_{1} S^{0}\right) \Delta A^{*} \cdot S^{0}-\left(k_{1} N^{0}+\frac{F^{\circ}}{V}\right) \Delta S^{*} \cdot S^{0}+ \\
\frac{1}{V}\left(C_{S 0}-S^{0}\right) \Delta F^{*} \cdot F^{0}+\frac{F^{\circ}}{V} \Delta C_{S 0}{ }^{*} \cdot S^{0} \\
\frac{d \Delta B^{*} \cdot S^{0}}{d t}=\left(k_{2} N^{0}\right) \Delta A^{*} \cdot S^{0}+\left(k_{2} A^{0}\right) \Delta N^{*} \cdot N^{0}- \\
\left(\frac{F^{\circ}}{V}\right) \Delta B^{*} \cdot S^{0}-\left(\frac{B^{0}}{V}\right) \Delta F^{*} \cdot F^{0}
\end{gathered}
$$

Accordingly, multiplying either by $V / F^{0} G^{0}$ or by $V / F^{0} N^{0}$, the next sets of equations are obtained:

$$
\begin{aligned}
& \frac{d \Delta A^{*}}{d t^{*}}=-\left(\frac{k_{2} N^{0} V}{F^{0}}+1\right) \Delta A^{*}+\left(\frac{V k_{1} N^{0}}{F^{0}}-\frac{V k_{2} A^{0} N^{0}}{F^{0} S^{0}}\right) \Delta N^{*}+ \\
& \frac{V k_{1} N^{0}}{F^{0}} \Delta S^{*}-\frac{A^{0}}{S^{0}} \Delta F^{*} \\
& \quad \frac{d \Delta N^{*}}{d t^{*}}=-\frac{V k_{2} S^{0}}{F^{0}} \Delta A^{*}-\left(\frac{V k_{1} S^{0}}{F^{0}}+\frac{V k_{2} A^{0}}{F^{0}}+1\right) \Delta N^{*}+ \\
& \frac{V k_{1} S^{0}}{F^{0}} \Delta S^{*}+\frac{\left(C_{N 0}-N^{0}\right)}{N^{0}} \Delta F^{*}+\Delta C_{N 0}{ }^{*} \\
& \frac{d \Delta G^{*}}{d t^{*}}=-\frac{V k_{1} G^{0}}{F^{0}} \Delta A^{*}-\left(\frac{V k_{1} N^{0}}{F^{0}}+1\right) \Delta S^{*}+\frac{\left(C_{S 0}-S^{0}\right)}{S^{0}} \Delta F^{*}+ \\
& \Delta C_{S 0^{*}}{ }^{*} \\
& \frac{d \Delta B^{*}}{d t^{*}}=\frac{V k_{2} N^{0}}{F^{0}} \Delta A^{*}+\frac{V k_{2} A^{0} N^{0}}{F^{0} S^{0}} \Delta N^{*}-\Delta B^{*}-\frac{B^{0}}{S^{0}} \Delta F^{*}
\end{aligned}
$$

where the term $t^{*}=\left(F^{\circ} \cdot t / V\right)$ implies a dimensionless parameter (dimensionless time). As a result, the system transforms into (33)

$$
\begin{aligned}
& \left(\begin{array}{c}
\frac{\mathrm{d} \Delta \mathrm{A}^{*}}{\mathrm{dt}^{*}} \\
\frac{\mathrm{d} \Delta \mathrm{N}^{*}}{\mathrm{dt}^{*}} \\
\frac{\mathrm{d} \Delta \mathrm{S}^{*}}{\mathrm{dt}^{*}} \\
\frac{\mathrm{d} \Delta \mathrm{B}^{*}}{\mathrm{dt}^{*}}
\end{array}\right) \\
& =\left(\begin{array}{cccc}
-\left(\frac{\mathrm{Vk}_{2} \mathrm{~N}^{0}}{\mathrm{~F}^{\circ}}+1\right) & \frac{\mathrm{Vk}_{1} \mathrm{~N}^{0}}{\mathrm{~F}^{0}}-\frac{\mathrm{Vk}_{2} \mathrm{~A}^{0} \mathrm{~N}^{0}}{\mathrm{~F}^{0} \mathrm{~S}^{0}} & \frac{\mathrm{Vk}_{1} \mathrm{~N}^{0}}{\mathrm{~F}^{\circ}} & 0 \\
-\left(\frac{\mathrm{Vk}_{2} \mathrm{~S}^{0}}{\mathrm{~F}^{\circ}}\right) & -\left(\frac{\mathrm{Vk}_{1} \mathrm{~S}^{0}+\mathrm{Vk}_{2} \mathrm{~A}^{0}+\mathrm{F}^{\circ}}{\mathrm{F}^{\circ}}\right) & \left(\frac{\mathrm{Vk}_{1} \mathrm{~S}^{0}}{\mathrm{~F}^{\circ}}\right) & 0 \\
-\left(\frac{\mathrm{Vk}_{1} \mathrm{~S}^{0}}{\mathrm{~F}^{\circ}}\right) & -\left(\frac{\mathrm{Vk}_{1} \mathrm{~N}^{0}}{\mathrm{~F}^{\circ}}+1\right) & 0 & 0 \\
\left(\frac{\mathrm{Vk}_{2} \mathrm{~N}^{0}}{\mathrm{~F}^{\circ}}\right) & \left(\frac{\mathrm{Vk}_{2} \mathrm{~A}^{0} \mathrm{~N}^{0}}{\mathrm{~F}^{\circ} \mathrm{S}^{0}}\right) & 0 & -1
\end{array}\right)\left(\begin{array}{l}
\Delta \mathrm{A}^{*} \\
\Delta \mathrm{N}^{*} \\
\Delta \mathrm{S}^{*} \\
\Delta \mathrm{B}^{*}
\end{array}\right)+ \\
& \left(\begin{array}{ccc}
-\frac{A^{0}}{S^{0}} & 0 & 0 \\
\frac{\left(C_{\mathrm{N} 0}-\mathrm{N}^{0}\right)}{\mathrm{N}^{0}} & 0 & 1 \\
\frac{\left(\mathrm{C}_{\mathrm{S} 0}-\mathrm{G}^{0}\right)}{\mathrm{G}^{0}} & 1 & 0 \\
-\left(\frac{\mathrm{B}^{0}}{\mathrm{~S}^{0}}\right) & 0 & 0
\end{array}\right)\left(\begin{array}{c}
\Delta \mathrm{F}^{*} \\
\Delta \mathrm{C}_{\mathrm{S} 0} \\
\Delta \mathrm{C}_{\mathrm{N} 0}
\end{array}\right)
\end{aligned}
$$

Furthermore, as the transfer function G(s) is given by $G(s)=(s I-A)^{-1} B$, firstly it is necessary to calculate $(\mathrm{sI}-\mathrm{A})$. Therefore, we need to define the next elements of matrix $A$ :

$\mathrm{a}_{11}=\left(\frac{\mathrm{Vk}_{2} \mathrm{~N}^{0}}{\mathrm{~F}^{\circ}}+1\right) ; \mathrm{a}_{12}=\frac{\mathrm{Vk}_{1} \mathrm{~N}^{0}}{\mathrm{~F}^{0}}-\frac{\mathrm{Vk}_{2} \mathrm{~A}^{0} \mathrm{~N}^{0}}{\mathrm{~F}^{0} \mathrm{~S}^{0}} ; \mathrm{a}_{13}=\frac{\mathrm{Vk}_{1} \mathrm{~N}^{0}}{\mathrm{~F}^{\circ}}$ 


$$
\begin{aligned}
& \mathrm{a}_{21}=\left(\frac{\mathrm{Vk}_{2} \mathrm{~N}^{0}}{\mathrm{~F}^{\circ}}\right) ; \mathrm{a}_{22}=\left(\frac{\mathrm{Vk}_{1} \mathrm{~S}^{0}+\mathrm{Vk}_{2} \mathrm{~A}^{0}+\mathrm{F}^{\circ}}{\mathrm{F}^{\circ}}\right) ; \mathrm{a}_{23}=\left(\frac{\mathrm{Vk}_{1} \mathrm{~S}^{0}}{\mathrm{~F}^{\circ}}\right) \\
& \mathrm{a}_{31}=\left(\frac{\mathrm{Vk}_{1} \mathrm{~S}^{0}}{\mathrm{~F}^{\circ}}\right) ; \mathrm{a}_{32}=\left(\frac{\mathrm{Vk}_{1} \mathrm{~N}^{0}}{\mathrm{~F}^{\circ}}+1\right) \\
& \mathrm{a}_{41}=\left(\frac{\mathrm{Vk}_{2} \mathrm{~N}^{0}}{\mathrm{~F}^{\circ}}\right) ; \mathrm{a}_{42}=\left(\frac{\mathrm{Vk}_{2} \mathrm{~A}^{0} \mathrm{~N}^{0}}{\mathrm{~F}^{\circ} \mathrm{S}^{0}}\right) \\
& (\mathrm{sI}-\mathrm{A})=\left(\begin{array}{cccc}
\mathrm{s}+\mathrm{a}_{11} & -\mathrm{a}_{12} & -\mathrm{a}_{13} & 0 \\
\mathrm{a}_{21} & \mathrm{~s}+\mathrm{a}_{22} & -\mathrm{a}_{23} & 0 \\
\mathrm{a}_{31} & \mathrm{a}_{32} & \mathrm{~s} & 0 \\
-\mathrm{a}_{41} & -\mathrm{a}_{42} & 0 & \mathrm{~s}+1
\end{array}\right)
\end{aligned}
$$

In order to simulate the process, the next input parameter values are taken into account: $\mathrm{F}=0.1 \mathrm{~m}^{3} \mathrm{~s}^{-1}, C_{S O}=1$ $\mathrm{mol} \cdot \mathrm{m}^{-3}, C_{N O}=1 \mathrm{~mol} \cdot \mathrm{m}^{-3} k_{l}=0.32 \mathrm{~h}^{-1}, k_{2}=0.49 \mathrm{~h}^{-1}, k_{3}=0.27$ $\mathrm{h}^{-1}, \mathrm{~V}=1 \mathrm{~m}^{3}, N=0.2 \mathrm{~mol} \cdot \mathrm{m}^{-3}, S=0.3 \mathrm{~mol} \cdot \mathrm{m}^{-3}, A=0.5$ $\mathrm{mol} \cdot \mathrm{m}^{-3}, B=0.2 \mathrm{~mol} \cdot \mathrm{m}^{-3}$. As a result, the respective transfer functions are given by the following set of equations:

From input $u_{l}=F$ to output

$$
\begin{aligned}
& y 1=A: G(s)=\frac{-1.6667 \mathrm{~s}^{3}-11.49 \mathrm{~s}^{2}-12.2919 \mathrm{~s}-2.4619}{\mathrm{~s}^{4}+7.39 \mathrm{~s}^{3}+15.8504 \mathrm{~s}^{2}+12.8288 \mathrm{~s}+3.3684} \\
& \mathrm{y} 2=\mathrm{N}: \mathrm{G}(\mathrm{s})=\frac{4 \mathrm{~s}^{3}+16.61 \mathrm{~s}^{2}+18.8436 \mathrm{~s}+6.2336}{\mathrm{~s}^{4}+7.39 \mathrm{~s}^{3}+15.8504 \mathrm{~s}^{2}+12.8288 \mathrm{~s}+3.3684} \\
& \mathrm{y} 3=\mathrm{S}: \mathrm{G}(\mathrm{s})=\frac{2.3333 \mathrm{~s}^{3}+12.2833 \mathrm{~s}^{2}+20.7807 \mathrm{~s}+10.8307}{\mathrm{~s}^{4}+7.39 \mathrm{~s}^{3}+15.8504 \mathrm{~s}^{2}+12.8288 \mathrm{~s}+3.3684} \\
& \mathrm{y} 4=\mathrm{B}: \mathrm{G}(\mathrm{s})=\frac{-0.6667 \mathrm{~s}^{3}+0.64 \mathrm{~s}^{2}+4.656 \mathrm{~s}+5.2533}{\mathrm{~s}^{4}+7.39 \mathrm{~s}^{3}+15.8504 \mathrm{~s}^{2}+12.8288 \mathrm{~s}+3.3684}
\end{aligned}
$$

from input $u_{2}=C_{S 0}$ to output

$$
\begin{aligned}
& \mathrm{y} 1=\mathrm{A}: \mathrm{G}(\mathrm{s})=\frac{0.6400 \mathrm{~s}^{2}+2.5088 \mathrm{~s}+1.8688}{\mathrm{~s}^{4}+7.39 \mathrm{~s}^{3}+15.8504 \mathrm{~s}^{2}+12.8288 \mathrm{~s}+3.3684} \\
& \mathrm{y} 2=\mathrm{N}: \mathrm{G}(\mathrm{s})=\frac{0.9600 \mathrm{~s}^{2}+1.9200 \mathrm{~s}+0.96}{\mathrm{~s}^{4}+7.39 \mathrm{~s}^{3}+15.8504 \mathrm{~s}^{2}+12.8288 \mathrm{~s}+3.3684} \\
& \mathrm{y} 3=\mathrm{S}: \mathrm{G}(\mathrm{s})=\frac{1 \mathrm{~s}^{3}+7.39 \mathrm{~s}^{2}+13.6616 \mathrm{~s}+7.2716}{\mathrm{~s}^{4}+7.39 \mathrm{~s}^{3}+15.8504 \mathrm{~s}^{2}+12.8288 \mathrm{~s}+3.3684} \\
& \mathrm{y} 4=\mathrm{B}: \mathrm{G}(\mathrm{s})=\frac{2.1952 \mathrm{~s}+3.3994}{\mathrm{~s}^{4}+7.39 \mathrm{~s}^{3}+15.8504 \mathrm{~s}^{2}+12.8288 \mathrm{~s}+3.3684} \\
& \text { from input } u_{3}=C_{N 0} \text { to output } \\
& \mathrm{y} 1=\mathrm{A}: \mathrm{G}(\mathrm{s})=\frac{-0.9933 \mathrm{~s}^{2}-2.0429 \mathrm{~s}-1.0496}{\mathrm{~s}^{4}+7.39 \mathrm{~s}^{3}+15.8504 \mathrm{~s}^{2}+12.8288 \mathrm{~s}+3.3684} \\
& \mathrm{y} 2=\mathrm{N}: \mathrm{G}(\mathrm{s})=\frac{\mathrm{s}^{3}+2.98 \mathrm{~s}^{2}+2.5944 \mathrm{~s}+0.6144}{\mathrm{~s}^{4}+7.39 \mathrm{~s}^{3}+15.8504 \mathrm{~s}^{2}+12.8288 \mathrm{~s}+3.3684} \\
& \mathrm{y} 3=\mathrm{S}: \mathrm{G}(\mathrm{s})=\frac{-1.64 \mathrm{~s}^{2}-3.9336 \mathrm{~s}-2.2936}{\mathrm{~s}^{4}+7.39 \mathrm{~s}^{3}+15.8504 \mathrm{~s}^{2}+12.8288 \mathrm{~s}+3.3684} \\
& \mathrm{y} 4=\mathrm{B}: \mathrm{G}(\mathrm{s})=\frac{1.6333 \mathrm{~s}^{2}+2.2605 \mathrm{~s}-0.0251}{\mathrm{~s}^{4}+7.39 \mathrm{~s}^{3}+15.8504 \mathrm{~s}^{2}+12.8288 \mathrm{~s}+3.3684}
\end{aligned}
$$

Finally, the inverse Laplace transformation leads to equations (165) to (176)

$$
\text { input } u_{l}=F
$$

$$
\begin{aligned}
& \mathrm{y}_{1}(\mathrm{t})=0.723 \mathrm{e}^{-4.4235 \mathrm{t}}-3.0361 \mathrm{e}^{-1.4364 \mathrm{t}}+ \\
& 0.6464 \mathrm{e}^{-0.5301 \mathrm{t}} \\
& \mathrm{y}_{2}(\mathrm{t})=2.4697 \mathrm{e}^{-4.4235 \mathrm{t}}+1.3396 \mathrm{e}^{-1.4364 \mathrm{t}} \\
& 0.1907 \mathrm{e}^{-0.5301 \mathrm{t}} \\
& \mathrm{y}_{3}(\mathrm{t})=1.0725 \mathrm{e}^{-4.4235 \mathrm{t}}-0.4997 \mathrm{e}^{-1.4364 \mathrm{t}}+ \\
& 1.7604 \mathrm{e}^{-0.5301 \mathrm{t}} \\
& \mathrm{y}_{4}(\mathrm{t})=-1.3854 \mathrm{e}^{-4.423 \mathrm{t}}+1.8044 \mathrm{e}^{-1.436 \mathrm{t}}- \\
& 3.0969 \mathrm{e}^{-\mathrm{t}}+2.0111 \mathrm{e}^{-0.53 \mathrm{t}} \\
& \text { input } \mathrm{u}_{2}=\mathrm{C}_{\mathrm{S} 0}: \\
& \mathrm{y}_{1}(\mathrm{t})=0.0827 \mathrm{e}^{-4.4235 \mathrm{t}}-0.3507 \mathrm{e}^{-1.4364 \mathrm{t}}+ \\
& 0.4335 \mathrm{e}^{-0.5301 \mathrm{t}} \\
& \mathrm{y}_{2}(\mathrm{t})=-0.2826 \mathrm{e}^{-4.4235 \mathrm{t}}+0.1548 \mathrm{e}^{-1.4364 t}+ \\
& 0.1278 \mathrm{e}^{-0.5301 \mathrm{t}} \\
& \mathrm{y}_{3}(\mathrm{t})=-0.1227 \mathrm{e}^{-4.4235 \mathrm{t}}-0.0577 \mathrm{e}^{-1.4364 \mathrm{t}}+ \\
& 1.1804 \mathrm{e}^{-0.5301 \mathrm{t}} \\
& \mathrm{y}_{4}(\mathrm{t})=0.1585 \mathrm{e}^{-4.423 \mathrm{t}}+0.2084 \mathrm{e}^{-1.436 \mathrm{t}}- \\
& 1.7154 \mathrm{e}^{-\mathrm{t}}+1.3485 \mathrm{e}^{-0.5301 \mathrm{t}}
\end{aligned}
$$

$$
\begin{aligned}
& \text { input } \mathrm{u}_{3}=\mathrm{C}_{\mathrm{N} 0}: \\
& \mathrm{y}_{1}(\mathrm{t})=0.2876^{-4.4235 \mathrm{t}}-0.1393 \mathrm{e}^{-1.4364 t}- \\
& 0.1482 \mathrm{e}^{-0.5301 \mathrm{t}} \\
& \mathrm{y}_{2}(\mathrm{t})=0.98226 \mathrm{e}^{-4.4235 \mathrm{t}}+0.0615 \mathrm{e}^{-1.4364 t}- \\
& 0.0437 \mathrm{e}^{-0.5301 \mathrm{t}} \\
& \mathrm{y}_{3}(\mathrm{t})=0.4266 \mathrm{e}^{-4.4235 \mathrm{t}}-0.0229 \mathrm{e}^{-1.4364 t}- \\
& 0.4036 \mathrm{e}^{-0.5301 \mathrm{t}} \\
& \mathrm{y}_{4}(\mathrm{t})=-0.550 \mathrm{e}^{-4.4235 \mathrm{t}}+0.082 \mathrm{e}^{-1.4364 t}+ \\
& 0.929 \mathrm{e}^{-\mathrm{t}}-0.461 \mathrm{e}^{-0.5301 \mathrm{t}}
\end{aligned}
$$

The response to a step function for the input $\mathrm{u}_{1}$ is presented as an example in Fig. 2,

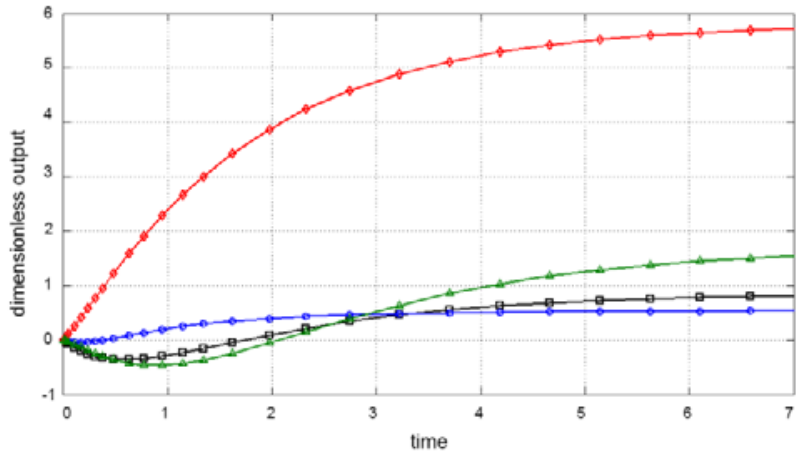

Fig. 2: Step response for the system of glycerol oxidation with $\mathrm{NO}_{2}$. Input $u_{l}=F$ to respective outputs $\mathrm{y}_{1}=\mathrm{A} \square ; \mathrm{y}_{2}=\mathrm{N} \mathrm{o} ; \mathrm{y}_{3}=\mathrm{S} \diamond ; \mathrm{y}_{4}=\mathrm{B} \Delta$

\section{Conclusions}

Today, special attention is paid to partial oxidation of several sugar alcohols because it gives oxidation intermediate products with high utility value. However, a major problem of partial oxidation of these compounds lies in its low reactivity and high oxidation reactivity of intermediates, which is expressed by different values of the rate constants of oxidation in the first step of the reaction and in subsequent steps of the oxidation reaction. The yield depends on the ratio of rate constants. For simulation purposes, specified constants and initial concentrations of nitrous oxide and reactant can be specified.

This work was supported by the Ministry of Education, Youth and Sports of the Czech Republic within the National Sustainability Programme project No. LO1303 (MSMT7778/2014).

\section{References}

1. B. Ohtani, S.-I. Takamiya, Y. Hirai, M. Sudoh, S.-I. Nishimoto, and T. Kagiya, J. Chem. Soc. Perkin Trans, 2, 175-179, [1992].

2. H. S. Fogler, Elements of Chemical Reaction Engineering, (Prentice Hall, New Jersey, 1999).

3. B. A. Finlayson, Introduction to Chemical Engineering Computing. (John Wiley and Sons, Inc, 2006). 
4. I. Tosun, Modeling in Transport Phenomena, (Elsevier, 2007).

5. R. L. Burden and J. D. Faires, Numerical Methods, (Brooks Cole, 2002).

6. A. S. Foust, L. A. Wenzel, C. W. Clump, L. Maus, and L. B. Andersen, Principles of unit operations, (John Wiley \& Sons, Inc, New Delhi, 1980).

7. G. Stephanopoulos, Chemical Process Control: An Introduction to Theory and Practice. (Prentice-Hall, 1984).

8. E. D. Seborg, T. F. Edgar, and D. Mellichamp, Process Dynamics and Control. (John Wiley and Sons, Inc., 1989).

9. R. Aris, Elementary Chemical Reactor Analysis. (Dover Publications, Boston 2000). 